\title{
Low T-cell subsets prior to development of virus-associated cancer in HIV-seronegative men who have sex with men
}

\author{
Anupriya Dutta ${ }^{1} \cdot$ Hajime Uno ${ }^{2} \cdot$ David R. Lorenz $^{1}$ - Steven M. Wolinsky ${ }^{3}$ Dana Gabuzda ${ }^{1}$
}

Received: 23 March 2018 / Accepted: 5 October 2018 / Published online: 12 October 2018

(c) The Author(s) 2018

\begin{abstract}
Immunological parameters that influence susceptibility to virus-associated cancers in HIV-seronegative individuals are unclear. We conducted a case-control cohort study of immunological parameters associated with development of incident virus-associated cancers among 532 HIV-seronegative men who have sex with men (MSM) enrolled in the Multicenter AIDS Cohort Study (MACS) with median (IQR) 21 (8-26) years of follow-up. Thirty-two incident virus-associated cancers (anal cancer, non-Hodgkin lymphoma, liver cancer, other cancers with etiologies linked to human papillomavirus, Epstein-Barr virus, hepatitis B virus, or human herpesvirus-8) were identified among 3,408 HIV-seronegative men in the MACS during 1984-2010. Cases were matched for demographics, smoking, and follow-up to 500 controls without cancer. Mixed-effects and Cox regression models were used to examine associations between nadir or recent CD4, CD8, and white blood cell (WBC) counts or CD4:CD8 ratios and subsequent diagnosis of virus-associated cancers. Men with incident virus-associated cancers had lower CD4 and WBC counts over a 6-year window prior to diagnosis compared to men without cancer ( $p=0.001$ and 0.03 , respectively). Low CD4 cell count and nadir, CD4 count-nadir differential, and CD4:CD8 ratio nadir were associated with increased 2-year risk of incident virus-associated cancers in models adjusted for demographics and smoking (hazard ratios $1.2-1.3$ per 100 or 0.1 unit decrease, respectively; $p<0.01$ ). Other associated factors included heavy smoking and past or current hepatitis B virus infection. These findings show that low CD4 cell counts, CD4 nadir, and CD4:CD8 cell ratios are independent predictors for subsequent risk of virus-associated cancers in HIV-seronegative MSM.
\end{abstract}

Keywords Cancer risk $\cdot$ Oncogenic viruses $\cdot \mathrm{HBV} \cdot \mathrm{CD} 4 \mathrm{~T}$ cells $\cdot \mathrm{CD} 8 \mathrm{~T}$ cells $\cdot$ Lymphopenia

\section{Introduction}

Viral infections are likely etiological agents in nearly onefifth of cancer cases [1-3]. Virus-associated cancers with causes linked to oncogenic viruses include genital, anal, and

Electronic supplementary material The online version of this article (https://doi.org/10.1007/s10552-018-1090-4) contains supplementary material, which is available to authorized users.

Dana Gabuzda

dana_gabuzda@dfci.harvard.edu

1 Department of Cancer Immunology and Virology, Dana Farber Cancer Institute, Center for Life Science 1010, 450 Brookline Avenue, Boston, MA 02215, USA

2 Department of Medical Oncology, Dana-Farber Cancer Institute, Boston, MA 02215, USA

3 Division of Infectious Diseases, Department of Medicine, Northwestern University Feinberg School of Medicine, Chicago, IL 60611, USA oral cancers (HPV), non-Hodgkin and Hodgkin lymphomas (EBV), Kaposi sarcoma (HHV-8), and liver cancer (HBV). However, the oncogenic potential of viruses such as HPV, $\mathrm{EBV}, \mathrm{HHV}-8$, and $\mathrm{HBV}$ is challenging to ascertain due to the multifactorial nature of cancer development [3, 4]. Pinpointing viral origins of cancers is complicated by several factors, including prolonged viral latency before tumors arise, involvement of host and environmental co-factors in tumorigenesis, and host variability in natural immune mechanisms that protect against tumor development $[1,2]$.

The immune response to infection by oncogenic viruses is a key factor that helps to explain why only a fraction of these viral infections result in tumors [2, 5]. Immunosuppression and inflammation are co-factors for viral carcinogenesis [2, $6,7]$, and cancers with viral etiologies occur more frequently in immunosuppressed populations, including HIV-infected and transplant patients [6, 8-14]. Primary immunodeficiencies such as idiopathic CD4+ lymphopenia (CD4 T-cell count $<300$ cells $/ \mu \mathrm{l}$ ) and idiopathic neutropenia (neutrophil 
count $<200$ cells $/ \mu \mathrm{l}$ ) are rare disorders that have been associated with elevated risk of virus-associated cancers [15]. Cytopenias can also occur in patients with cirrhosis [16, 17], while subclinical immune dysfunction has been associated with liver fibrosis [18]. Immunological parameters predictive for development of virus-associated cancers and their relationship to liver diseases in the general population are unclear.

The prognostic value of immunological factors in determining treatment responses underscores their importance for cancer immunotherapies [6, 19], and suggests that natural immune protection and immunosurveillance can influence cancer susceptibility. However, few studies have evaluated immunologic risk profiles associated with development of specific types of virus-associated cancers with etiologies linked to HPV, EBV, HBV, HCV, or HHV-8 in HIV-seronegative individuals [6]. Here, we conducted a longitudinal matched case-control study of HIV-seronegative men who have sex with men (MSM) in the MACS (Multicenter AIDS Cohort Study) to evaluate immunological predictors of virus-associated cancers.

\section{Methods}

\section{Study cohort}

This is a matched case-control study of men enrolled in the MACS, an ongoing natural history cohort study of men who report sex with men. The MACS was established in 1984, enrolling 6,972 HIV-infected and HIV-uninfected MSM over three recruitment waves [1984-1985 ( $n=4,957), 1987-1991$ ( $n=665), 2001-2003(n=1,350)]$ across four study sites (Los Angeles, Chicago, Baltimore, and Pittsburgh). Behavioral, clinical, and laboratory data including HIV serology tests were collected at semi-annual visits as described [20, 21]. Institutional Review Boards at each study site approved the research, and all participants provided written informed consent.

Participants for this study were $532 \mathrm{HIV}$-seronegative men over age 18 followed between 1984 and 2010 with no pre-existing cancers and $\geq 2$ visits with values for immunological parameters. Thirty-two cases with incident virusassociated cancers were identified through a database search of cancer diagnoses among all HIV-seronegative participants in the MACS $(n=3,408)$. For cohort assembly, an initial search identified 25 incident virus-associated cancers that were matched 1:20 to 500 controls without cancer for age at endpoint, race, smoking, and calendar period using the MatchIT package in $\mathrm{R}$ version 3.2.4. We later identified seven additional incident cancers and examined whether case-control matching was still balanced after inclusion of these cases; demographics and smoking were still matched $(p>0.20)$ (Table 1), so we included these additional cases to increase precision of the analysis. HIV-negative serostatus was confirmed at study visits within 2 years of cancer diagnosis for 29 cases and within 4-5 years for three cases. Because all cases remained HIV-seronegative during followup, HIV RNA viral load assays were performed for only three cases; all were below the limit of detection of the assay ( $<50$ copies per $\mathrm{ml}$ of plasma).

\section{Data collection and risk factor classification}

The MACS public dataset (P23 release) was translated to a local SQL database and used for the analyses. Immunological parameters and clinical characteristics associated with cancer risk factors were censored at 1 year prior to last follow-up visit for both cases and controls to account for a potential lag between clinical diagnosis of incident cancer and date of cancer diagnosis ascertained through registry linkage. CD4 and CD8 T-cell counts, white blood cell (WBC) counts, CD4/CD8 ratios, and Fibrosis-4 (FIB-4) score (a non-invasive index of liver fibrosis calculated from age, aspartate aminotransferase (AST), alanine aminotransferase (ALT), and platelet count; FIB- $4<1.45$ has a negative predictive value of $90 \%$ for advanced fibrosis) [22] were summarized as time-varying variables using values nearest to 1 year before last follow-up visit. Nadir values were the lowest value during follow-up. Time-updated smoking was defined as smoking on average more than half a pack per day within last 6 years of follow-up. Hepatitis C virus (HCV) infection status was determined by positive $\mathrm{HCV}$ antibody or RNA test. HBV infection status was determined by positive Hepatitis B surface antigen (HBsAg) or e-antigen (HBeAg), or HBV DNA test.

\section{Cancer outcomes}

A total of 32 incident virus-associated cancers with etiologies linked to HPV, EBV, HHV-8, HBV, or HCV diagnosed during the study period were identified using International Classification of Diseases for Oncology, third edition (ICDO-3) codes. Incident cancers were ascertained continuously during follow-up using cancer registry linkage data, available medical records and death certificates, and self-reported cancer diagnoses [20]. After cancers were classified based on anatomical site and histology [20], analyses focused on a composite category, "virus-associated cancers," comprised of diagnoses associated with infection by HPV [anal cancer and head and neck squamous cell carcinomas (HNSCC)], EBV [non-Hodgkin lymphoma (NHL) and Hodgkin lymphoma (HL)], HHV-8 (Kaposi sarcoma [KS]), and HBV/ HCV (liver cancer). Despite an established oncogenic role of HPV in development of skin SCC [23], skin cancers were excluded from the analysis given potential confounding 
Table 1 Demographic and clinical characteristics by virus-associated cancer diagnosis

\begin{tabular}{|c|c|c|c|c|}
\hline & $\begin{array}{l}\text { All } \\
(n=532)\end{array}$ & $\begin{array}{l}\text { Controls } \\
(n=500)\end{array}$ & $\begin{array}{l}\text { Cases }^{\mathrm{a}} \\
(n=32)\end{array}$ & $p$ \\
\hline Cumulative person years, median (IQR) & $21(8-26)$ & $22(8-26)$ & $15(8-21)$ & 0.003 \\
\hline Age at entry visit, median (IQR) & $35(30-42)$ & $35(30-42)$ & $42(36-45)$ & $<0.001$ \\
\hline Age at endpoint, median (IQR) & $54(47-61)$ & $54(47-61)$ & $57.5(49-62.2)$ & 0.22 \\
\hline Race & & & & 0.63 \\
\hline Non-black & $423(79.5)$ & $396(79.2)$ & $27(84.4)$ & \\
\hline Black & $109(20.5)$ & $104(20.8)$ & $5(15.6)$ & \\
\hline Heavy smoking ${ }^{\mathrm{b}}$ & & & & 0.37 \\
\hline No & $424(79.7)$ & $401(80.2)$ & $23(71.9)$ & \\
\hline Yes & $108(20.3)$ & $99(19.8)$ & $9(28.1)$ & \\
\hline Hepatitis $\mathrm{C}$ infection $^{\mathrm{c}}$ & $43(8.1)$ & $42(8.4)$ & $1(3.1)$ & 0.47 \\
\hline Hepatitis B infection $^{\mathrm{c}}$ & $22(4.4)$ & $15(3.0)$ & 7 (21.9) & $<0.001$ \\
\hline \multicolumn{5}{|l|}{ Sexually transmitted infection ${ }^{\mathrm{c}}$} \\
\hline Genital warts & $168(31.6)$ & $160(32)$ & $8(25)$ & 0.53 \\
\hline Syphilis & $74(13.9)$ & $68(13.6)$ & $6(18.8)$ & 0.58 \\
\hline $\mathrm{CD} 4$ count $(\text { cells } / \mu \mathrm{l})^{\mathrm{d}}$ & & & & 0.32 \\
\hline$<200$ & $0(0)$ & $0(0)$ & $0(0)$ & \\
\hline $200-349$ & $3(0.6)$ & $3(0.6)$ & $0(0)$ & \\
\hline $350-499$ & $17(3.2)$ & $14(2.8)$ & $3(9.7)$ & \\
\hline $500-599$ & $44(8.3)$ & $42(8.4)$ & $2(6.5)$ & \\
\hline$\geq 600$ & 467 (87.9) & $441(88.2)$ & $26(83.9)$ & \\
\hline CD 4 count $(\text { cells } / \mu \mathrm{l})^{\mathrm{d}}$, median $(\mathrm{IQR})$ & $943(729-1,158)$ & $947.5(736-1,162)$ & $858(693-1,019)$ & 0.15 \\
\hline CD4 nadir $<500(\text { cells } / \mu \mathrm{l})^{\mathrm{c}}$ & $152(28.6)$ & $137(27.4)$ & $15(46.9)$ & $\mathbf{0 . 0 3}$ \\
\hline CD4 nadir $(\text { cells } / \mu \mathrm{l})^{\mathrm{c}}$, median $(\mathrm{IQR})$ & $614(483-771)$ & $620(484-775)$ & $512(370-652)$ & 0.007 \\
\hline CD8 count (cells/ $\mu \mathrm{l})^{\mathrm{d}}$, median $(\mathrm{IQR})$ & $515(381-693)$ & $512(383-685)$ & $629(378-759)$ & 0.32 \\
\hline CD8 nadir $<250(\text { cells } / \mu \mathrm{l})^{\mathrm{c}}$ & $152(28.6)$ & $141(28.2)$ & $11(34.4)$ & 0.58 \\
\hline CD8 nadir $(\text { cells } / \mu \mathrm{l})^{\mathrm{c}}$, median $(\mathrm{IQR})$ & $323.5(237-410)$ & $326(238-410)$ & $304(232-386)$ & 0.69 \\
\hline CD4:CD8 ratio ${ }^{\mathrm{d}}$, median (IQR) & $1.84(1.3-2.4)$ & $1.85(1.4-2.4)$ & $1.62(1.2-2.1)$ & 0.08 \\
\hline $\mathrm{CD} 4: \mathrm{CD} 8$ ratio $<1^{\mathrm{d}}$ & $44(8.3)$ & $39(7.8)$ & $5(16.1)$ & 0.20 \\
\hline CD4:CD8 ratio nadir ${ }^{\mathrm{c}}$, median (IQR) & $1.2(0.93-1.5)$ & $1.2(0.94-1.6)$ & $0.92(0.72-1.4)$ & 0.001 \\
\hline $\mathrm{CD} 4: \mathrm{CD} 8$ ratio nadir $<1^{\mathrm{c}}$ & $164(30.8)$ & $146(29.2)$ & $18(56.2)$ & 0.003 \\
\hline White blood cell (WBC) count $(\text { cells/ } \mu \mathrm{l})^{\mathrm{d}}$, median (IQR) & $6,000(5,000-7,500)$ & $6,050(5,000-7,500)$ & $5,700(5,000-7,400)$ & 0.56 \\
\hline WBC nadir $(\text { cells } / \mu \mathrm{l})^{\mathrm{c}}$, median $(\mathrm{IQR})$ & $4,400(3,800-5,300)$ & $4,400(3,800-5,300)$ & $4,300(3,400-5,225)$ & 0.35 \\
\hline Cumulative sexual partners ${ }^{\mathrm{e}} \geq 10$ partners & $289(54.3)$ & $272(54.4)$ & $17(54.8)$ & 1.00 \\
\hline Median years to diagnosis from CD4 nadir & & & $9.75(6.0-14.8)$ & \\
\hline Median years to diagnosis from CD8 nadir & & & $8.5(4.5-14.5)$ & \\
\hline Median years to diagnosis from $\mathrm{CD} 4: \mathrm{CD} 8$ ratio nadir & & & $8.17(4.5-13.5)$ & \\
\hline Median years to diagnosis from WBC nadir & & & $7.25(3.4-13.5)$ & \\
\hline
\end{tabular}

Data are $n(\%)$ unless otherwise indicated

Bold values indicate $p<0.05$

${ }^{a}$ Composite measure of first virus-associated cancer diagnosis of Kaposi sarcoma (KS), non-Hodgkin lymphoma (NHL), Hodgkin lymphoma (HL), anal cancer, head and neck squamous cell carcinoma (HNSCC), or liver cancer

${ }^{\mathrm{b}} 0.5$ packs/day for more than half the duration of follow-up

${ }^{\mathrm{c}}$ Anytime following enrollment to study endpoint

${ }^{\mathrm{d}}$ Nearest available value 1 year prior to endpoint

${ }^{\mathrm{e}}$ Summarized over first three visits

effects of ultra-violet exposure, which was not ascertained. ICD-O-3 site codes for anal cancers were 21.0-21.8 and
19.9-20.9 based on a prior study in the MACS cohort [21]. ICD-O-3 site codes for HNSCC cases were 2.9, 4.9, and 
10.2. The NHL cases were B-cell lymphomas (four diffuse large B-cell lymphomas, two follicular lymphomas, two malignant lymphomas of skin and unknown site, one mantle cell lymphoma, one chronic lymphocytic leukemia).

\section{Statistical analysis}

Univariate or bivariate tests were conducted using Wilcoxon Rank-Sum test, paired $t$ test, Pearson Chi-Square, or analysis of variance (ANOVA) for cohort characteristics by cancer diagnosis and CD4 count nadir. Exploratory analyses of longitudinal data utilized plots of mean values of immunological parameters for cases and controls during last 10 years of follow-up. Linear mixed-effects models were fit for longitudinal CD4 and WBC count as continuous dependent variables over the last 6 years of follow-up to determine the association between incident viral cancer diagnoses with these parameters and their rates of change. Observation time was left-truncated at 6 years prior to diagnosis because 428 participants $(81.8 \%)$ had at least 12 semi-annual visits with immunological data, while data coverage became sparser going back further in time (Supplemental Material 1). Log2 transformation was applied to WBC values to improve normality of distributions. Models were adjusted for baseline age (at 6 years prior to follow-up), race, heavy smoking, FIB-4 $>1.45$, and time to diagnosis; all models included random intercepts and slopes. Cox regression models were used to calculate adjusted hazard ratios (HR) of incident virus-associated cancer diagnoses associated with timevarying 2-year lagged CD4 cell count and CD4:CD8 ratio, CD4 count-nadir differential, and CD4 count and CD4:CD8 ratio nadirs during follow-up. We used a stepwise approach to evaluate Cox regression models, screening for predictors among these immunological parameters to evaluate their associations with risk. Modeling started with age, race, smoking, and CD4 count, followed by sequentially replacing the CD4 count variable with CD4 nadir, CD4:CD8 ratio, or CD4:CD8 ratio nadir. We hypothesized that including both CD4 count and CD4 count minus CD4 nadir in the model would provide a more dynamic representation of the relationship between low CD4 counts and cancer risk and therefore we extended models 1 and 2 by including both CD4 count minus CD4 nadir among the parameters tested. Models were adjusted for cumulative heavy smoking ( $\geq 0.5$ packs/day average in last 6 years of follow-up) and race; age was used as the time scale. All analyses were performed using $\mathrm{R}$ version 3.2 .

\section{Results}

\section{Cohort characteristics}

The study population of 32 virus-associated cancer cases and 500 matched controls had a median (IQR) age and follow-up of 35 (30-42) and 21 (8-26) years, respectively (Table 1). The majority of incident cancers were anal cancers and NHL ( $n=9$ cases each) followed by liver cancer ( $n=6$ cases), while incident HL, HNSCC, and KS accounted for three cases or less (Supplemental Material 2). Virus-associated cancers comprised the first diagnosis among two of four subjects with multiple cancer diagnoses (Supplemental Material 3 ); in the remaining two cases, virus-associated cancers were the second primary malignancy, diagnosed 17 and 2 years after previous diagnoses of thyroid cancer and prostate cancer, respectively. Compared to controls, virus-associated cancer cases were slightly older at entry, with shorter followup and higher percentage with HBV infection $(p \leq 0.003)$. Cases were similar to controls with regard to race, smoking, calendar period, HCV infection, number of sexual partners, and sexually transmitted infections (Table 1). Cases had a non-significant trend toward lower median CD4 cell counts compared to controls (858 vs. 947 cells $/ \mu$ l, respectively; $p=0.15$ ), while CD4 nadir was significantly lower (512 vs. 620 cells $/ \mu \mathrm{l} ; p=0.007$ ) and percentage with CD4 nadir $<500$ cells $/ \mu$ l was higher ( $46.9 \%$ vs. $27.4 \% ; p=0.03$ ). There was a trend toward lower median CD4:CD8 ratio nadir in cases compared to controls (1.62 vs. 1.85, respectively; $p=0.08$ ), while the percentage with CD4:CD8 ratio nadir $<1$ was higher $(56.2 \%$ vs. $29.2 \% ; p=0.003)$. Median time from CD4, CD4:CD8, CD8, and WBC nadir to cancer diagnosis ranged from 7.25 to 9.75 years.

\section{Immunological parameters prior to cancer diagnosis}

Immunological parameters were first examined in exploratory analyses of individual and mean trajectories in cases and controls during follow-up, which indicated an optimal window between 6 and 10 years prior to diagnosis for evaluation of prognostic markers based on group differences and data coverage (Supplemental Material 1 and Figs. 1, 2, 3). Longitudinal trends of $\mathrm{CD} 4, \mathrm{CD} 8$, and $\mathrm{WBC}$ counts in individual virus-associated cancer cases showed variable patterns over 10 years or more prior to diagnosis, including chronically low counts for all three parameters, chronically low CD4 and WBC counts only, and declining CD4 and WBC counts; representative cases with immunological lab data available over 15 years or longer are shown in Fig. 1. The distribution of number of visits with immunological lab data for all cases and controls in the study cohort showed that $100 \%$, $81.8 \%$, and $63 \%$ of participants had immunological lab data 

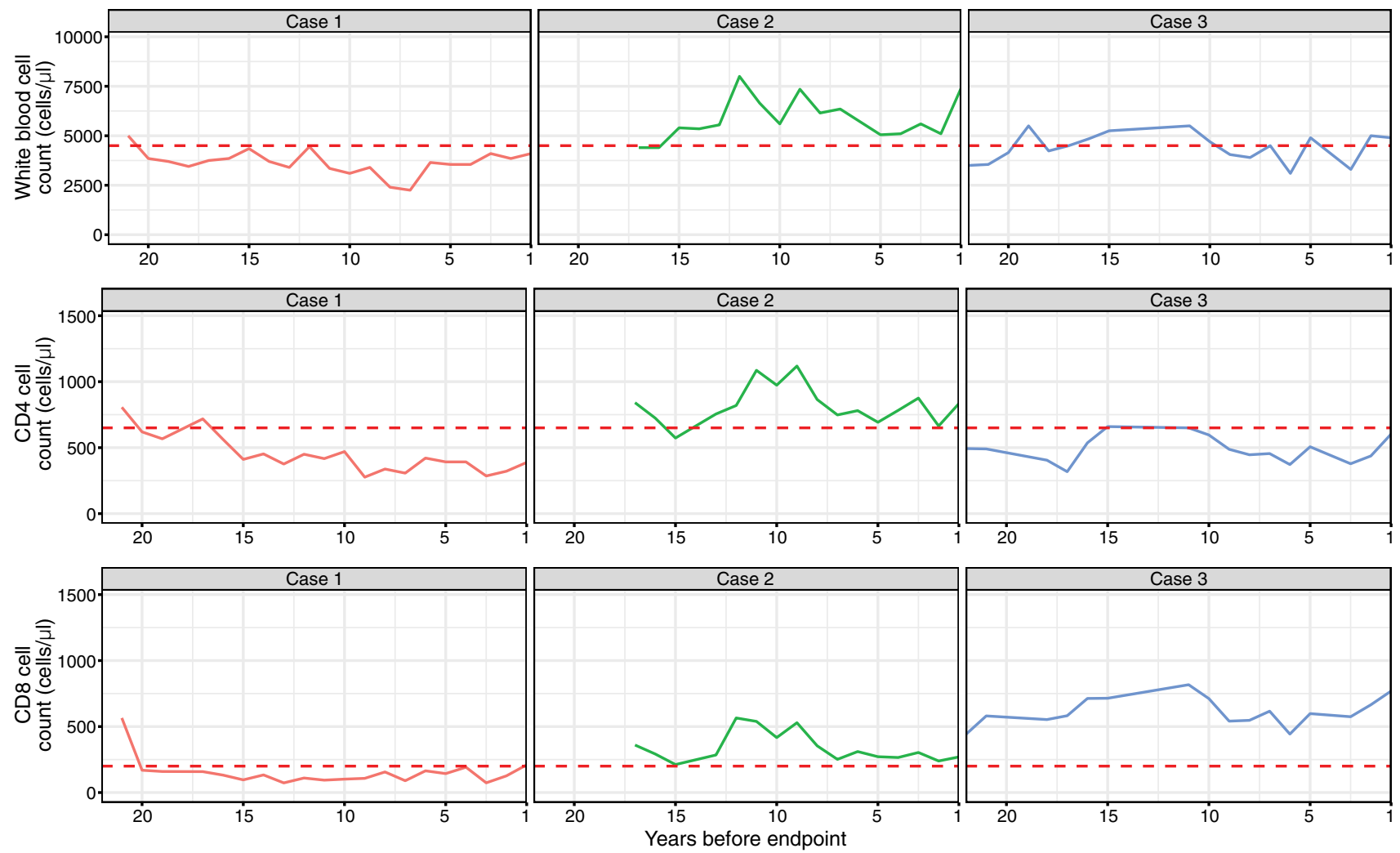

Fig. 1 Longitudinal trends of immune parameters in three representative virus-associated cancer cases with immunological lab data available over 15 years or longer. Liver cancer, anal cancer, and Kaposi sarcoma were diagnosed in cases 1,2 , and 3, respectively. Dashed

for at least 2,12, and 20 visits, respectively, corresponding to 1,6 , and 10 years of follow-up (Supplemental Material 1). Given this distribution of data coverage, exploratory analyses evaluated prognostic markers over 10 years for groups by cancer status and 6 years for groups by individual diagnoses. These exploratory analyses showed lower mean trajectories for immunological parameters, including CD4, $\mathrm{CD} 8$, WBC counts, and CD4:CD8 ratios, in cases compared to controls over a 10 year window (Fig. 2). When examined by individual diagnoses, subjects who developed NHL had slightly higher mean CD4, CD8, and WBC trajectories compared to those who developed solid-tissue tumors and controls (Fig. 3).

\section{Clinical characteristics associated with low CD4 counts}

Next, we sought to examine clinical characteristics associated with low CD4 nadir in the study cohort. Although CD4 lymphopenia is often idiopathic, previous studies showed that medical conditions associated with CD4 lymphopenia in HIV-negative individuals include cirrhosis and other advanced liver disease [16-18]. Therefore, we examined liver lines indicate lower reference range thresholds for normal values $(4,500,650$, and 200 cells/ $\mu \mathrm{l}$ for $\mathrm{WBC}, \mathrm{CD} 4$, and CD8 counts, respectively)

disease-related factors in groups by CD4 nadir $<500$ cells/ $\mu \mathrm{l}$ (Table 2). The frequencies of HCV and HBV infection, heavy alcohol consumption, liver conditions, and cirrhosis did not differ between groups by CD4 nadir. However, HBV infection and cirrhosis were more common among cases compared to controls, regardless of CD4 nadir status. The proportion with FIB-4 scores $>1.45$, a common threshold indicating liver fibrosis in HCV infection [22], was nearly twice as high in groups with CD4 count nadir $<500$ cells/ $\mu \mathrm{l}$ compared to those with CD4 nadir $\geq 500$ cells $/ \mu 1$ among both cases and controls $(62.5 \%$ vs. $37.5 \%$, and $34.4 \%$ vs. $20.3 \%$, respectively). Platelet counts below the normal adult reference range $\left(<150 \times 10^{9} / l\right)$, a common finding in patients with advanced liver disease, were also more common in cases and controls with CD4 nadir $<500$ cells/ $\mu$ l compared to corresponding groups with $\mathrm{CD} 4$ nadir $\geq 500$ cells $/ \mu 1$ (53.3\% vs. $17.6 \%, p=0.001$, and $32.8 \%$ vs. $17.9 \%, p=0.08$, respectively).

Next, we sought to determine if additional immunological parameters that could influence virus-associated cancer risk were detected among subjects with low CD4 nadir. To address this question, we evaluated total $\mathrm{T}$ - and $\mathrm{B}$-cell and white blood cell counts in groups by CD4 nadir $<500$ 

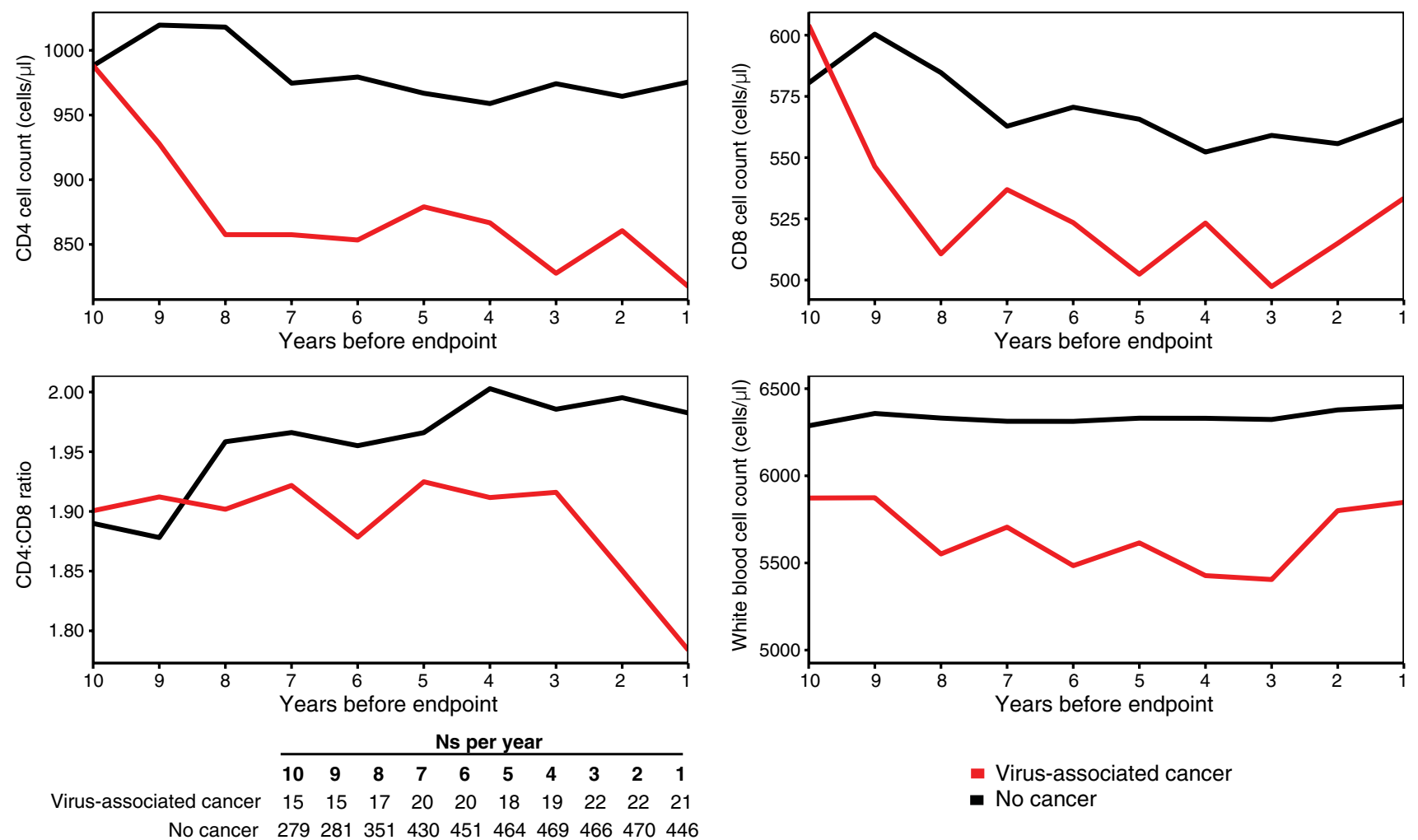

Fig. 2 Mean longitudinal trajectories of immune parameters in groups by virus-associated cancer diagnosis. One subject was censored due to chronic use of antiviral medication for more than 10 years

cells/ $\mu \mathrm{l}$. Total B-cell and T-cell count nadir and median summaries were determined for each subject, and summarized by cancer diagnosis (Table 2). Total B-cell count nadir and median were lower among cases and controls with CD4 nadir $<500$ cells/ $\mu$ l compared to those with CD4 nadir $\geq 500$ cells $/ \mu \mathrm{l}(p=0.004$ and 0.02 for cases; $p<0.001$ and $p<0.001$ for controls, respectively). Trends for WBC and total T-cell counts paralleled those of CD4 cell counts; controls with CD4 nadir $<500$ cells/ $\mu$ had more than twice the percentage of subjects with WBC nadir $<4,000$ cells/ $\mu \mathrm{l}$, and nearly twofold lower mean T-cell count nadir, compared to corresponding groups with CD4 nadir $\geq 500$ cells/ $\mu \mathrm{l}(p=0.002)$.

Given that prolonged antibiotic use has been linked to alterations in immune defenses [24-26], we examined selfreported duration of antibiotic use during follow-up. Antibiotic use was reported at more visits in cases and controls with CD4 nadir $<500$ cells/ $\mu$ compared to those with CD4 nadir $\geq 500$ cells/ $\mu 1$ among cases and controls (median 5 vs. 8 visits, $p=0.003$, and 3 vs. 8 visits, $p=0.03$, respectively). However, we did not observe a trend of antibiotic use prior to $\mathrm{CD} 4$ nadir.

\section{Mixed-effects models}

Given differences in CD4 and WBC counts in Figs. 2 and 3 , we investigated the association of CD4 and WBC counts with incident virus-associated cancers within a 6-year time window in mixed-effects models. An incident diagnosis of virus-associated cancer was associated with lower CD4 cell and white blood cell counts in models adjusted for age, race, heavy smoking, and FIB-4 scores $>1.45(p=0.001$ and 0.032, respectively; Fig. 4 and Supplemental Material 4). High FIB-4 was independently associated with lower CD4 and white blood cell counts, while heavy smoking was associated with higher counts (Supplemental Material 4).

\section{Cox models}

To examine the prognostic value of CD4 and CD8 T-cell parameters relative to incident virus-associated cancers, Cox regression models were fit to CD4 cell count, CD4 cell count-nadir differential, and CD4:CD8 ratio and nadir with adjustments for age (as the time scale), race, and heavy smoking (Table 3). Lower CD4 cell count nadir showed a strong association with risk of incident virusassociated cancers (adjusted HRs/100 cells/ $\mu$ l decrease [95\% CI] $1.31[1.13,1.51] ; p<0.001)$. Current CD4 cell 

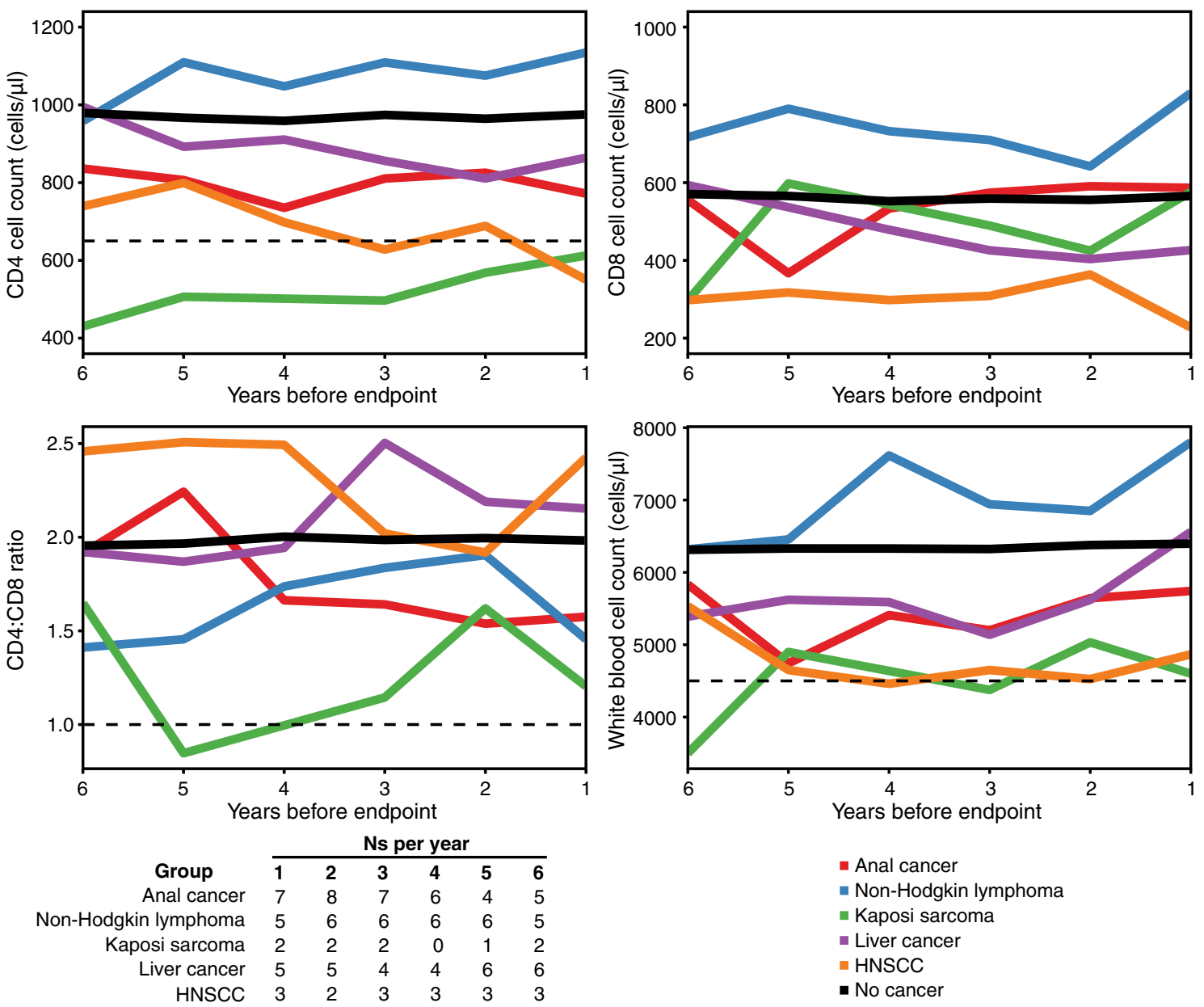

Fig. 3 Mean longitudinal trajectories of immune parameters in groups by type of virus-associated cancer. Hodgkin lymphoma cases $(n=3)$ were excluded due to limited data. Dashed lines indicate

lower reference range thresholds for normal values $(4,500,650$, and 200 cells/ $\mu$ for WBC, CD4, and CD8 counts, respectively)

count-nadir differential was a better predictor of incident virus-associated cancers (adjusted HRs/100 cells/ $\mu \mathrm{l}$ decrease and 95\% CI $1.27[1.09,1.48], p=0.002)$ than recent CD4 count (adjusted HRs/100 cells/ $\mu$ l decrease [95\% CI] 1.17 [1.03-1.33]; $p=0.014)$. Current CD4:CD8 ratios were not associated with increased risk of virus-associated cancers $(p=0.179)$; however, nadir CD4:CD8 ratio was associated with a three to fourfold increased risk of virus-associated cancers (adjusted HRs, 0.1 unit decrease and 95\% CI 1.18 $[1.06,1.31] ; p=0.002)$. Heavy smoking was associated with a three to fourfold increased risk of virus-associated cancers, while race had no significant associations in these models (Table 3).

\section{Discussion}

In this longitudinal case-control study of HIV-uninfected men with median 21 years of follow-up, low CD4 T-cell count and nadir and low CD4:CD8 ratio nadir were associated with increased risk of virus-associated cancers ( $p \leq 0.002$ in Cox hazard models). Our data suggest these prognostic markers have predictive value for risk of virus-associated cancers over a 6- to 8-year window prior to diagnosis; a subset of cases had chronically low CD4 and/or low CD8 counts as long as 10 years or longer prior to diagnosis (Fig. 1). In Cox hazard models, low CD4 cell count and nadir were associated with increased hazard of virus-associated cancers (adjusted HRs [95\% CIs] 1.17 [1.03-1.33], and 1.3 [1.13-1.5], respectively). Low CD4 count-nadir differential (adjusted HR [95\% CI] $1.27[1.09-1.48]$ ) was a stronger predictor of incident virus-associated cancers than recent CD4 count. Low 
Table 2 Immunological and liver disease parameters by CD4 nadir and virus-associated cancer status

\begin{tabular}{|c|c|c|c|c|c|c|}
\hline & \multicolumn{3}{|l|}{ Controls $(n=500)$} & \multicolumn{3}{|l|}{ Cases $(n=32)$} \\
\hline & $\begin{array}{l}\text { CD4 nadir } \geq 500 \\
\text { cells/ } \mu \mathrm{l}(n=363)\end{array}$ & $\begin{array}{l}\text { CD4 nadir }<500 \\
\text { cells/ } \mu \mathrm{l}(n=137)\end{array}$ & $p$ & $\begin{array}{l}\mathrm{CD} 4 \text { nadir } \geq 500 \\
\text { cells/ } \mu \mathrm{l}(n=17)\end{array}$ & $\begin{array}{l}\text { CD4 nadir }<500 \\
\text { cells/ } \mu \mathrm{l}(n=15)\end{array}$ & $p$ \\
\hline Hepatitis C (HCV) infection ${ }^{a}$ & $33(9.1)$ & $11(8.0)$ & 0.84 & $1(5.9)$ & $0(0.0)$ & 1.00 \\
\hline Hepatitis B (HBV) infection $^{a}$ & $8(2.2)$ & $7(5.1)$ & 0.16 & $3(17.6)$ & $4(26.7)$ & 0.85 \\
\hline HBV core antibody positive ${ }^{a}$ & $177(48.8)$ & $68(49.6)$ & 0.94 & $8(47.1)$ & $11(73.3)$ & 0.25 \\
\hline Heavy alcohol use ${ }^{b}$ & $88(24.8)$ & $32(24.4)$ & 1.00 & $5(31.2)$ & $4(26.7)$ & 1.00 \\
\hline Liver conditions $^{\mathrm{a}}$ & $23(6.3)$ & $9(6.6)$ & 1.00 & $1(5.9)$ & $3(20.0)$ & 0.50 \\
\hline Liver cirrhosis $^{\mathrm{a}}$ & $3(0.8)$ & $2(1.5)$ & 0.89 & $1(5.9)$ & $2(13.3)$ & 0.90 \\
\hline Fibrosis-4 (FIB-4) score $>1.45^{\mathrm{c}}$ & $72(20.3)$ & $44(34.4)$ & 0.002 & $3(37.5)$ & $5(62.5)$ & 0.61 \\
\hline FIB-4 score ${ }^{\mathrm{c}}$, median (IQR) & $0.96(0.76,1.21)$ & $1.11(0.87,1.47)$ & $<0.001$ & $1.28(0.99,2.82)$ & $1.42(1.22,2.05)$ & 0.67 \\
\hline Platelet count $<150\left(\times 10^{9} / 1\right)^{\mathrm{a}}$ & $65(17.9)$ & $45(32.8)$ & 0.001 & $3(17.6)$ & $8(53.3)$ & 0.08 \\
\hline WBC nadir $<4,000(\text { cells } / \mu \mathrm{l})^{\mathrm{a}}$ & $73(20.1)$ & $68(49.6)$ & $<\mathbf{0 . 0 0 1}$ & $3(17.6)$ & $9(60.0)$ & 0.035 \\
\hline B-cell count nadir $(\text { cells } / \mu \mathrm{l})^{\mathrm{a}}$, mean $(\mathrm{sd})$ & $361.4(145.26)$ & $258.7(108.96)$ & $<\mathbf{0 . 0 0 1}$ & $385.0(128.89)$ & $241.3(80.67)$ & 0.001 \\
\hline Median B-cell count $(\text { cells } / \mu \mathrm{l})^{\mathrm{c}}$, mean $(\mathrm{sd})$ & $569.2(193.27)$ & $484.5(164.20)$ & $<0.001$ & $628.8(204.31)$ & $432.3(145.61)$ & 0.004 \\
\hline T-cell count nadir $(\text { cells } / \mu \mathrm{l})^{\mathrm{a}}$, mean $(\mathrm{sd})$ & $1,124(264)$ & $667(219)$ & $<0.001$ & $1,185(427)$ & $712(268)$ & 0.001 \\
\hline Median T-cell count $(\text { cells } / \mu \mathrm{l})^{\mathrm{c}}$, mean $(\mathrm{sd})$ & $1,634(378)$ & $1,290(355)$ & $<\mathbf{0 . 0 0 1}$ & $1,671(480)$ & $1,219(356)$ & 0.006 \\
\hline Visits with antibiotics use $^{\mathrm{a}}$, median (IQR) & $5.00(2.00,11.00)$ & $8.00(3.00,14.00)$ & 0.003 & $3.00(1.00,7.00)$ & $8.00(3.50,13.00)$ & 0.03 \\
\hline Antibiotic use at $\geq 4$ visits $^{\mathrm{a}}$ & $211(58.1)$ & $98(71.5)$ & 0.008 & $7(41.2)$ & $11(73.3)$ & 0.14 \\
\hline
\end{tabular}

Data are $n(\%)$ unless otherwise indicated

Bold values indicate $p<0.05$

${ }^{a}$ Anytime following enrollment to study endpoint

${ }^{b} \geq 14$ drinks/week, or $\geq 5$ drinks on one occasion at least monthly

${ }^{\mathrm{c}}$ Nearest available value 1 year prior to endpoint

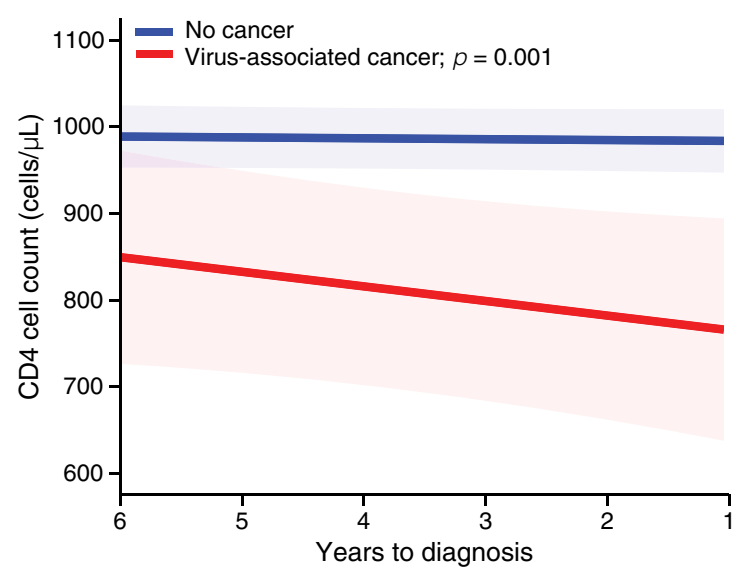

Fig. 4 Mean longitudinal trajectories of CD4 and white blood cell counts in groups via mixed-effect models for virus-associated cancer vs. no cancer. Given discordant patterns for CD4 and WBC counts in NHL cases compared to other virus-associated cancers (see Fig. 3),

CD4:CD8 ratio nadir was also associated with increased hazard of virus-associated cancer (adjusted HR [95\% CI] 1.18 [1.06-1.31]), while recent CD4:CD8 ratio did not show a significant association. The role of CD4 and CD8 $\mathrm{T}$ cells in immune control of viral infections is well established. CD4 cells are necessary for maintenance of CD8 cell functions [27], while depletion of CD4 T cells leads

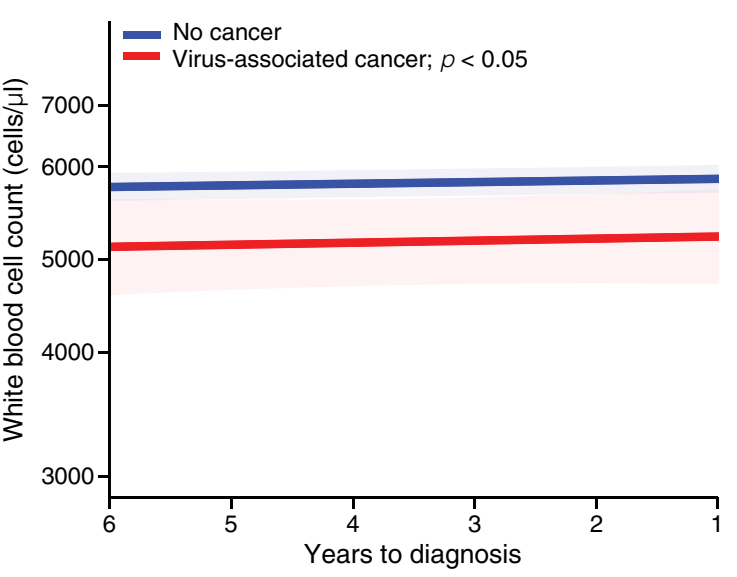

NHL cases were excluded from these models. Full models adjusted for age, race, smoking, FIB-4 score, and time to diagnosis are shown in Supplemental Material 4

to CD8 T-cell exhaustion. Low CD4:CD8 ratios correlate with immune activation in virally suppressed HIVinfected individuals, and have prognostic significance for AIDS-related morbidity including some viral cancers [28]. Together, these findings underscore the importance of CD4 T cells in natural immune protection against viral cancers in healthy HIV-seronegative people. 
Table 3 Association of CD4 cell count or CD4:CD8 ratio and nadirs with virus-associated cancer diagnosis

\begin{tabular}{|c|c|c|}
\hline & \multicolumn{2}{|c|}{ Virus-associated cancer $^{\mathrm{a}}$} \\
\hline & $\operatorname{HR}(95 \% \mathrm{Cl})$ & $p$ \\
\hline \multicolumn{3}{|l|}{ Model 1} \\
\hline CD4 count (per 100 cells/ $\mu$ l decrease) & $1.17(1.03,1.33)$ & 0.014 \\
\hline Heavy smoking ${ }^{\mathrm{b}}$ & $4.54(1.82,11.29)$ & 0.001 \\
\hline Black race & $1.08(0.38,3.09)$ & 0.881 \\
\hline \multicolumn{3}{|l|}{ Model 2} \\
\hline CD4 nadir (per 100 cells/ $\mu$ l decrease) & $1.31(1.13,1.51)$ & $<\mathbf{0 . 0 0 1}$ \\
\hline Heavy smoking ${ }^{\mathrm{b}}$ & $4.05(1.65,9.95)$ & 0.002 \\
\hline Black race & $1.34(0.47,3.82)$ & 0.579 \\
\hline \multicolumn{3}{|l|}{ Model 3} \\
\hline CD4 count (per 100 cells/ $\mu$ l decrease) & $1.36(1.15,1.62)$ & $<\mathbf{0 . 0 0 1}$ \\
\hline CD4 count minus CD4 nadir (per 100 cells/ $\mu 1$ decrease) & $1.27(1.09,1.48)$ & 0.002 \\
\hline Heavy smoking ${ }^{\mathrm{b}}$ & $4.79(1.82,12.59)$ & 0.002 \\
\hline Black race & $1.22(0.42,3.55)$ & 0.709 \\
\hline \multicolumn{3}{|l|}{ Model 4} \\
\hline CD4:CD8 ratio (per 0.1 unit decrease) & $1.04(0.98,1.1)$ & 0.179 \\
\hline Heavy smoking ${ }^{\mathrm{b}}$ & $3.23(1.35,7.74)$ & 0.009 \\
\hline Black race & $1.17(0.39,3.48)$ & 0.778 \\
\hline \multicolumn{3}{|l|}{ Model 5} \\
\hline $\mathrm{CD} 4: \mathrm{CD} 8$ ratio nadir (per 0.1 unit decrease) & $1.18(1.06,1.31)$ & 0.002 \\
\hline Heavy smoking ${ }^{a}$ & $4.08(1.55,10.73)$ & 0.004 \\
\hline Black race & $1.19(0.36,3.93)$ & 0.777 \\
\hline
\end{tabular}

Multivariate Cox proportional hazards models adjusted for age

Bold values indicate $p<0.05$

${ }^{a}$ Composite measure of first virus-associated cancer diagnosis of Kaposi sarcoma, non-Hodgkin lymphoma, Hodgkin lymphoma, anal cancer, head and neck squamous cell carcinoma, or liver cancer $(n=32$ cases)

${ }^{\mathrm{b}} 0.5$ packs/day or more on average over last 6 years of follow-up
Low CD4 cell counts could not be attributed to specific medical conditions, nor use of immunosuppressive drugs, in most virus-associated cancer cases in the study cohort. Twenty-nine subjects (4 cases and 25 controls) met criteria for idiopathic CD4 lymphopenia, defined by CD4 cell count $<300$ for at least one visit. One liver cancer case was lymphophenic at multiple visits, with CD4 and CD8 cell counts $<300$ and 100, respectively (Fig. 1, left panels); these observations together with our finding that FIB- $4>1.45$ is more frequent among subjects with CD4 nadir $<500$ compared to those with CD4 nadir $\geq 500$ are consistent with other studies linking lymphopenias with advanced liver disease $[16,18]$. Lower platelet counts in groups with CD4 nadir $<500$ may also reflect liver disease in some subjects. Antibiotic use was more frequent among cases and controls with CD4 nadir $<500$ compared to subjects that maintained CD4 nadir $\geq 500$. The significance of this finding remains unclear, but it could reflect increased susceptibility to bacterial infections in the setting of low CD4 counts or microbiome changes that influence immune function [24, 26, 29].
Distinct immunological profiles and trends were observed for subjects that developed NHL compared to those that developed solid-tissue cancers (Fig. 3), which may reflect differences in the natural history of EBV infection and EBV-related cancer compared to other oncogenic virus infections. Greater than $90 \%$ of the general population is exposed to EBV infection by their mid-twenties [4]; in addition to immunosuppression, host co-factors, oncogenic hits, and virus re-activation from latency play a role in development of EBV-related cancers [2]. The relative increase in mean CD4 and CD8 T-cell count trajectories prior to diagnosis in NHL cases compared to controls (Fig. 3) may reflect lymphocytosis driven by EBV-induced B-cell proliferation [30]. However, previous studies suggest that B-cell activation markers (e.g., sCD27, sCD30) and immunodeficiency markers (e.g., low $\mathrm{CD} 4$ and low CD4:CD8 ratio) represent different classes of early detection markers, linked to NHL through distinct mechanisms $[8,31]$.

The study cohort had a high prevalence $(52.8 \%)$ of participants with positive tests for Hepatitis B core protein 
antibodies (anti-HBc), an indicator of past HBV infection, consistent with studies demonstrating higher rates of HBV in MSM compared to the general population [32]. All subjects with liver cancer had evidence of past or current HBV or HCV infection. Prior studies demonstrated lower CD4 cell counts in HIV-infected individuals positive for anti$\mathrm{HBc}$ compared to $\mathrm{HBc}$ seronegatives [33]; in our cohort, the percentage of cases positive for anti-HBc among those with CD4 nadir $<500$ cells/ $\mu$ l was higher than that of cases with CD4 nadir $\geq 500$ cells $/ \mu$ l ( $73.3 \%$ vs. $47.1 \%$ ), raising the possibility that prior HBV infection may impact immune functions that protect against some non-hepatic viral cancers [16-18]. Chronic HBV infection has been associated with T-cell exhaustion, but the association between anti-HBc positivity and T-cell exhaustion is unclear [34]. The impact of past or current HBV infection on risk of non-hepatic viral cancers is an open question that warrants further study.

To our knowledge, this is the first case-control study to examine immunological predictors of incident virusassociated cancer in a cohort of HIV-seronegative men. We acknowledge limitations of our study, however. The relatively small number of cancer outcomes and sample size limited adjustment for a large number of potential confounding factors through regression models and statistical power to detect some associations. Some cohort characteristics are more prevalent in MSM compared to the general population, including smoking, $\mathrm{HBV} / \mathrm{HCV}$ infection, liver dysfunction, and sexually transmitted infections, which limits generalizability of our findings. For example, smoking has been associated with elevated risk of HNSCC and anal cancers after controlling for HPV-infection status and other factors, and could influence some findings [35-37]. Our analysis was limited by lack of data for other immune cell types, limiting our ability to evaluate findings within a broader immunological context. Although HIV serology testing was performed at semi-annual visits, three cancer cases lacked HIV serology testing within 4-5 years prior to cancer diagnosis so we cannot exclude the possibility that these individuals might have seroconverted between their last study visit and cancer diagnosis. We right-censored immunological data 1 year prior to cancer diagnosis and therefore cannot reach conclusions about immunological parameters at time of diagnosis. Lastly, serological testing and other lab data for EBV, HPV, and HHV-8 detection was not available, so we cannot reach any conclusions regarding infections with an oncogenic virus and development of virus-associated cancers.

In summary, our study shows that HIV-seronegative MSM with low nadir CD4 counts or low CD4:CD8 ratio nadirs have elevated risk of developing virus-associated cancers within a 6-year time window. Furthermore, our studies suggest that past or current HBV infection coupled with low CD4 count or nadir or low CD4:CD8 ratio is a profile associated with increased risk of developing virus-associated cancers in otherwise healthy HIV-seronegative men. Such individuals might be candidates to screen for treatable oncogenic viral infections or pre-malignant lesions to reduce their cancer risk.

Acknowledgments Data for this manuscript were obtained by the Multicenter AIDS Cohort Study (MACS) with centers at Baltimore (U01-AI35042): The Johns Hopkins University Bloomberg School of Public Health: Joseph B. Margolick (PI), Jay Bream, Todd Brown, Barbara Crain, Adrian Dobs, Richard Elion, Richard Elion, Michelle Estrella, Lisette Johnson-Hill, Sean Leng, Anne Monroe, Cynthia Munro, Michael W. Plankey, Wendy Post, Ned Sacktor, Jennifer Schrack, Chloe Thio; Chicago (U01-AI35039): Feinberg School of Medicine, Northwestern University, and Cook County Bureau of Health Services: Steven M. Wolinsky (PI), John P. Phair, Sheila Badri, Dana Gabuzda, David Ostrow, Frank J. Palella, Jr., Sudhir Penugonda, Susheel Reddy, Matthew Stephens, Linda Teplin; Los Angeles (U01-AI35040): University of California, UCLA Schools of Public Health and Medicine: Roger Detels (PI), Otoniel Martínez-Maza (Co-PI), Aaron Aronow, Peter Anton, Robert Bolan, Elizabeth Breen, Anthony Butch, Shehnaz Hussain, Beth Jamieson, Eric N. Miller, John Oishi, Harry Vinters, Dorothy Wiley, Mallory Witt, Otto Yang, Stephen Young, Zuo Feng Zhang; Pittsburgh (U01-AI35041): University of Pittsburgh, Graduate School of Public Health: Charles R. Rinaldo (PI), Lawrence A. Kingsley (Co-PI), James T. Becker, Phalguni Gupta, Kenneth Ho, Susan Koletar, Jeremy J. Martinson, John W. Mellors, Anthony J. Silvestre, Ronald D. Stall; Data Coordinating Center (UM1AI35043): The Johns Hopkins University Bloomberg School of Public Health: Lisa P. Jacobson (PI), Gypsyamber D’Souza (Co-PI), Alison, Abraham, Keri Althoff, Jennifer Deal, Priya Duggal, Sabina Haberlen, Alvaro Muoz, Derek Ng, Janet Schollenberger, Eric C. Seaberg, Sol Su, Pamela Surkan. Website located at http://www.statepi.jhsph.edu/macs/ macs.html. Cancer incidence data were provided by the following state agencies: (1) Maryland Cancer Registry, Center for Cancer Prevention and Control, Department of Health and Mental Hygiene, Baltimore, MD 21201; (2) Illinois Department of Public Health, Illinois State Cancer Registry; (3) Bureau of Health Statistics \& Research, Pennsylvania Department of Health, Harrisburg, Pennsylvania; (4) Ohio Cancer Incidence Surveillance System (OCISS), Ohio Department of Health $(\mathrm{ODH})$, a cancer registry partially supported in the National Program of Cancer Registries at the Centers for Disease Control and Prevention (CDC) through Cooperative Agreement \# 5U58DP000795-05; and (5) California Department of Public Health pursuant to California Health and Safety Code Section 103885; CDC's National Program of Cancer Registries, under cooperative agreement 5NU58DP003862-04/ DP003862; the National Cancer Institute's Surveillance, Epidemiology and End Results Program under contract HHSN261201000140C awarded to the Cancer Prevention Institute of California, contract HHSN261201000035C awarded to the University of Southern California, and contract HHSN261201000034C awarded to the Public Health Institute. We acknowledge the State of Maryland, the Maryland Cigarette Restitution Fund, and the National Program of Cancer Registries of the CDC for the funds that support the collection and availability of the cancer registry data. The analyses, findings, interpretations, and conclusions of this report are those of the authors. No endorsement by any of the states providing data, the National Cancer Institute, the CDC, or their Contractors and Subcontractors is intended nor should be inferred. The authors also acknowledge Dr. Isaac Solomon for helpful discussions of primary data and Elizabeth Carpelan for assistance with manuscript preparation.

Author contributions AD participated in study design, performed data assembly and statistical analysis, drafted the manuscript, and prepared tables and figures. HU participated in study design and statistical 
analysis. DL participated in data parsing, assembly, and analysis. SW participated in study design, data review, and data analysis. DG conceived of the study, supervised its design, coordination, assembly, and analysis, and helped write and edit the manuscript. All authors read, participated in editing the manuscript, and approved the final manuscript.

Funding This work was supported by NIH Grants to D.G. (DP1 DA028994, R01 DA30985, and R01 DA40391). The work was also supported in part by NIH funding to the Northwestern University Clinical Research Unit of the MACS (U01-AI35039), with additional co-funding from the National Cancer Institute (NCI), National Institute on Drug Abuse (NIDA), and National Institute of Mental Health (NIMH). A.D. was supported in part by NIH T32-AG000222 and T32-AI007386. Biostatistical consultation was provided by the NIHfunded Harvard Catalyst (UL1 TR001102). The MACS is funded by the National Institute of Allergy and Infectious Diseases (NIAID) [U01-AI35039, U01-AI35040; U01-AI35041; U01-AI35042; and UM1-AI35043], with additional co-funding from the National Cancer Institute (NCI), National Institute on Drug Abuse (NIDA), and National Institute of Mental Health (NIMH) at the National Institutes of Health (NIH). MACS data collection is also supported by UL1-TR000424 (JHU CTSA).

\section{Compliance with ethical standards}

Conflict of interest The authors report no conflicts of interest.

Open Access This article is distributed under the terms of the Creative Commons Attribution 4.0 International License (http://creativeco mmons.org/licenses/by/4.0/), which permits unrestricted use, distribution, and reproduction in any medium, provided you give appropriate credit to the original author(s) and the source, provide a link to the Creative Commons license, and indicate if changes were made.

\section{References}

1. Butel JS (2000) Viral carcinogenesis: revelation of molecular mechanisms and etiology of human disease. Carcinogenesis 21:405-426

2. Mesri EA, Feitelson MA, Munger K (2014) Human viral oncogenesis: a cancer hallmarks analysis. Cell Host Microbe 15:266-282

3. Morales-Sanchez A, Fuentes-Panana EM (2014) Human viruses and cancer. Viruses 6:4047-4079

4. White MK, Pagano JS, Khalili K (2014) Viruses and human cancers: a long road of discovery of molecular paradigms. Clin Microbiol Rev 27:463-481

5. Corthay A (2014) Does the immune system naturally protect against cancer? Front Immunol 5:197

6. Michaud DS, Houseman EA, Marsit CJ, Nelson HH, Wiencke JK, Kelsey KT (2015) Understanding the role of the immune system in the development of cancer: new opportunities for populationbased research. Cancer Epidemiol Biomarkers Prev 24:1811-1819

7. Moore PS, Chang Y (2017) Common commensal cancer viruses. PLoS Pathog 13:e1006078

8. Grulich AE, Vajdic CM, Cozen W (2007) Altered immunity as a risk factor for non-Hodgkin lymphoma. Cancer Epidemiol Biomarkers Prev 16:405-408

9. Grulich AE, van Leeuwen MT, Falster MO, Vajdic CM (2007) Incidence of cancers in people with HIV/AIDS compared with immunosuppressed transplant recipients: a meta-analysis. Lancet $370: 59-67$
10. Schulz TF (2009) Cancer and viral infections in immunocompromised individuals. Int J Cancer 125:1755-1763

11. Petrara MR, Giunco S, Serraino D, Dolcetti R, De Rossi A (2015) Post-transplant lymphoproliferative disorders: from epidemiology to pathogenesis-driven treatment. Cancer Lett 369:37-44

12. Duray A, Lacremans D, Demoulin S, Delvenne P, Saussez S (2014) Prognosis of HPV-positive head and neck cancers: implication of smoking and immunosuppression. Adv Cell Mol Otolaryngol 2:25717

13. Hoover R, Fraumeni JF Jr (1973) Risk of cancer in renal-transplant recipients. Lancet 2:55-57

14. Biggar RJ, Chaturvedi AK, Goedert JJ, Engels EA, Study HACM (2007) AIDS-related cancer and severity of immunosuppression in persons with AIDS. J Natl Cancer Inst 99:962-972

15. Mortaz E, Tabarsi P, Mansouri D et al (2016) Cancers related to immunodeficiencies: update and perspectives. Front Immunol 7:365

16. McGovern BH, Golan Y, Lopez M et al (2007) The impact of cirrhosis on CD4+ T cell counts in HIV-seronegative patients. Clin Infect Dis 44:431-437

17. Albillos A, Lario M, Alvarez-Mon M (2014) Cirrhosis-associated immune dysfunction: distinctive features and clinical relevance. $\mathrm{J}$ Hepatol 61:1385-1396

18. Sipeki N, Antal-Szalmas P, Lakatos PL, Papp M (2014) Immune dysfunction in cirrhosis. World J Gastroenterol 20:2564-2577

19. Pitt JM, Vetizou M, Waldschmitt N et al (2016) Fine-tuning cancer immunotherapy: optimizing the gut microbiome. Cancer Res 76:4602-4607

20. Seaberg EC, Wiley D, Martinez-Maza O et al (2010) Cancer incidence in the multicenter AIDS Cohort Study before and during the HAART era: 1984 to 2007. Cancer 116:5507-5516

21. D'Souza G, Wiley DJ, Li X et al (2008) Incidence and epidemiology of anal cancer in the multicenter AIDS cohort study. J Acquir Immune Defic Syndr 48:491-499

22. Sterling RK, Lissen E, Clumeck N et al (2006) Development of a simple noninvasive index to predict significant fibrosis in patients with HIV/HCV coinfection. Hepatology 43:1317-1325

23. Karagas MR, Nelson HH, Sehr P et al (2006) Human papillomavirus infection and incidence of squamous cell and basal cell carcinomas of the skin. J Natl Cancer Inst 98:389-395

24. Ubeda C, Pamer EG (2012) Antibiotics, microbiota, and immune defense. Trends Immunol 33:459-466

25. Rajagopala SV, Vashee S, Oldfield LM et al (2017) The human microbiome and cancer. Cancer Prev Res (Phila) 10:226-234

26. Zitvogel L, Ayyoub M, Routy B, Kroemer G (2016) Microbiome and anticancer immunosurveillance. Cell 165:276-287

27. Matloubian M, Concepcion RJ, Ahmed R (1994) CD4+ T cells are required to sustain $\mathrm{CD} 8+$ cytotoxic $\mathrm{T}$-cell responses during chronic viral infection. J Virol 68:8056-8063

28. Serrano-Villar S, Sainz T, Lee SA et al (2014) HIV-infected individuals with low CD4/CD8 ratio despite effective antiretroviral therapy exhibit altered $\mathrm{T}$ cell subsets, heightened CD8+ T cell activation, and increased risk of non-AIDS morbidity and mortality. PLoS Pathog 10:e1004078

29. Ekmekciu I, von Klitzing E, Fiebiger U et al (2017) Immune responses to broad-spectrum antibiotic treatment and fecal microbiota transplantation in mice. Front Immunol 8:397

30. Cervera P, Guihot A, Gorochov G, Lassoued K, Coppo P (2015) Epstein-Barr virus-driven B Cell proliferation with CD4 + T cell expansion: a lymphomatoid granulomatosis-like disease related to hyperinterleukin-10 secretion of remarkably favourable outcome with rituximab. Scand J Immunol 82:532-538

31. Breen EC, Hussain SK, Magpantay L et al (2011) B-cell stimulatory cytokines and markers of immune activation are elevated several years prior to the diagnosis of systemic AIDS-associated 
non-Hodgkin B-cell lymphoma. Cancer Epidemiol Biomarkers Prev. 20:1303-1314

32. Mayer KH (2011) Sexually transmitted diseases in men who have sex with men. Clin Infect Dis 53(Suppl 3):S79-S83

33. Piroth L, Launay O, Michel ML et al (2016) Vaccination against Hepatitis B virus (HBV) in HIV-1-infected patients with isolated anti-HBV core antibody: the ANRS HB EP03 CISOVAC prospective study. J Infect Dis 213:1735-1742

34. Ye B, Liu X, Li X, Kong H, Tian L, Chen Y (2015) T-cell exhaustion in chronic hepatitis B infection: current knowledge and clinical significance. Cell Death Dis 6:e1694
35. Herrero R, Castellsague X, Pawlita M et al (2003) Human papillomavirus and oral cancer: the International Agency for Research on Cancer multicenter study. J Natl Cancer Inst 95:1772-1783

36. Daling JR, Madeleine MM, Johnson LG et al (2004) Human papillomavirus, smoking, and sexual practices in the etiology of anal cancer. Cancer 101:270-280

37. Helleberg M, Gerstoft J, Afzal S et al (2014) Risk of cancer among HIV-infected individuals compared to the background population: impact of smoking and HIV. AIDS 28:1499-1508 